\title{
Structure and Force Field of 1,3-Pentadiyne (Methyldiacetylene)
}

\author{
CLAUS J. NIELSEN and SVEIN SÆB $\emptyset$
}

Department of Chemistry, University of Oslo, Oslo 3, Norway

Microwave spectra of 1,3-pentadiyne-5,5,5- $d_{3}$ ([5,5,5-2 $\left.\mathrm{H}_{3}\right]-1,3$-pentadiyne), including four of the ${ }^{13} \mathrm{C}$ isotopic species, and 1,3-pentadiyne-5,5$d_{2}$ have been measured of the vibrational ground state. On the basis of the existing and the new spectroscopic data, the molecular structure $\left(r_{\mathrm{o}}\right.$, $\left.r_{\mathrm{m}}, r_{\mathrm{s}}\right)$ was derived and compared with results from $a b$ initio calculations.

The infrared vapour spectrum of 1,3-pentadiyne-5,5,5- $d_{3}$ was reinvestigated and the Coriolis coupling constant $\varsigma_{9}^{\mathrm{a}}$ was determined. Far infrared spectra of the parent molecule were measured and the heretofore unobserved vapour phase frequencies for the $v_{13}(e)$ and $v_{14}(e)$ modes obtained. A symmetry force field was derived from the vibrational and rotational spectroscopic data.

1,3-Pentadiyne (methyldiacetylene, later to be called MDA) has been subjected to only a few spectroscopic investigations. The microwave spectra of the vibrational ground state and of the first vibrational excited state of $v_{14}(e)$ have been studied for MDA and the three deuterated molecules: MDA-1- $d$, MDA-5,5,5- $d_{3}$ and MDA$d_{4}{ }^{1}$ This investigation was later extended to include a number of ${ }^{13} \mathrm{C}$ isotopes of the parent molecule and of the 1- $d$ species. ${ }^{2}$ From these data an $r_{0}$-structure assuming equal acetylenic bond lengths was proposed. ${ }^{2}$ More recently, the microwave spectra of the vibrational excited states, $v_{13}$, $v_{14}, 2 v_{14}$ and $3 v_{14}$, have been studied in greater detail ${ }^{3}$ leading to very accurate rovibronic parameters.

The vibrational spectra of MDA, MDA-1- $d$, MDA-5,5,5- $d_{3}$ and MDA- $d_{4}$ have been reported. ${ }^{4}$ The vibrational spectrum af $\mathrm{MDA},{ }^{5}$ including the Coriolis coupling constants for the degenerate methyl vibrations, and a normal coordinate analysis ${ }^{6}$ were reported from this laboratory.

\section{EXPERIMENTAL}

Small samples of MDA and MDA-5,5,5- $d_{3}$ were collected as "intermediates" in the synthesis of 2,4-hexadiyne (dimethyldiacetylene), 2,4-hexadiyne-1,1,1- $d_{3}$ and 2,4-hexadiyne- $d_{6}{ }^{{ }^{4}}$ The isotopic purity of MDA-5,5,5- $d_{3}$, checked by mass spectrometry, was ca. $97 \%$.

Microwave spectra were recorded on a Hewlett-Packard 8460A MRR spectrometer. Sample temperatures varied from -40 to $-60^{\circ} \mathrm{C}$ at pressures less than 0.01 Torr. Frequencies were measured to an accuracy of $0.05 \mathrm{MHz}$.

Infrared gas phase spectra were measured using a $10 \mathrm{~cm}$ cell (CsI windows) and a $20 \mathrm{~cm}$ cell (polyethylene windows) on a Bruker IFS 114C Fourier transform spectrometer.

\section{MICROWAVE SPECTRA}

The rotational spectra of MDA are those of a strictly symmetric top molecule with a positive value of $D_{\mathrm{JK}}$. A number of strong lines due to vibrational excited states were observed but not studied in any detail. The spectrum of MDA-5,5$d_{2}$, measured as an "isotopic impurity", is that of an extremely near prolate rotor with only the $K=1$ lines split.

Derived rotational parameters for MDA-5,5,5$d_{3}$, four of the ${ }^{13} \mathrm{C}$ isotopic species and MDA-5,5$d_{2}$ are presented in Table 1 . In all cases the rotational constant and $D_{\mathrm{J}}$ are strongly correlated $(0.9)$ and $D_{\mathrm{J}}$ poorly determined. A complete list of frequencies is available from the authors upon 
Table 1. Rotational parameters for 1,3-pentadiyne-5,5,5- $d_{3},{ }^{13} \mathrm{C}$ isotopic species and 1,3-pentadiyne$5,5-d_{2}$.

\begin{tabular}{llllll}
\hline Molecule & $B_{\mathrm{o}}[\mathrm{MHz}]$ & $D_{\mathrm{J}}[\mathrm{Hz}]$ & $D_{\mathrm{JK}}[\mathrm{kHz}]$ & $N_{\text {obs }}$ & rms $[\mathrm{MHz}]$ \\
\hline $\mathrm{D}_{3} \mathrm{CCCCCH}$ & $1834.8522(4)^{a}$ & $70.3(25)$ & $14.553(5)$ & 45 & 0.012 \\
$\mathrm{D}_{3}{ }^{13} \mathrm{CCCCCH}$ & $1795.8834(9)$ & $62(5)$ & $13.981(19)$ & 29 & 0.023 \\
$\mathrm{D}_{3} \mathrm{C}^{13} \mathrm{CCCCH}$ & $1828.2555(23)$ & $113(14)$ & $14.52(6)$ & 19 & 0.042 \\
$\mathrm{D}_{3} \mathrm{CCC}^{13} \mathrm{CCH}$ & $1818.587(3)$ & $81(17)$ & $14.26(5)$ & 16 & 0.042 \\
$\mathrm{D}_{3} \mathrm{CCCC} C^{13} \mathrm{CH}$ & $1785.0385(8)$ & $70(4)$ & $13.941(12)$ & 21 & 0.015 \\
$\mathrm{D}_{2} \mathrm{HCCCCCH}$ & $1899.3777(21)$ & $64 .(10)$ & $14.189(21)$ & 33 & 0.034 \\
& {$\left[C_{\mathrm{o}}=1891.3084(21)\right]$} & & & & \\
\hline
\end{tabular}

${ }^{a}$ Errors represent one standard deviation.

Table 2. Structure parameters for 1,3-pentadiyne (MDA) and butadiyne (DA).

\begin{tabular}{llllll}
\hline & $r_{\mathrm{o}}{ }^{a}$ & $r_{\mathrm{m}}$ & $r_{\mathrm{s}}$ & \multicolumn{2}{l}{$A b$ initio } \\
\cline { 5 - 6 } & & & & MDA & DA \\
\hline$r_{\mathrm{C}_{8}-\mathrm{H}_{9}}$ & $105.6(1)^{b}$ & $105.71(1)$ & $105.5(1)$ & 105.1 & 105.1 \\
$r_{\mathrm{C}_{\mathrm{C}} \equiv \mathrm{C}_{8}}$ & $120.8(1)$ & $120.86(1)$ & $120.9(1)$ & 119.3 & 119.3 \\
$r_{\mathrm{C}_{6}-\mathrm{C}_{7}}$ & $137.6(2)$ & $137.22(1)$ & $137.5(4)$ & 137.9 & 137.8 \\
$r_{\mathrm{C}_{5} \equiv \mathrm{C}_{6}}$ & $120.9(2)$ & $120.82(2)$ & $120.8(4)$ & 119.4 & 119.3 \\
$r_{\mathrm{C}_{4}-\mathrm{C}_{5}}$ & $145.4(1)$ & $145.43(1)$ & $145.6(3)$ & 146.5 & \\
$r_{\mathrm{C}_{4}-\mathrm{H}_{1}}$ & $110.7(1)$ & $110.70(2)$ & $110.5(1)$ & 108.1 & \\
$a_{\mathrm{C}_{5} \mathrm{C}_{4} \mathrm{H}_{1}}$ & $110.32(3)$ & $110.46(1)$ & $110.35(5)$ & 110.6 & \\
\hline
\end{tabular}

${ }^{a}$ Distances in pm. ${ }^{b}$ Numbers in parantheses represent the standard deviation.

request or from NBS, ${ }^{*}$ where it has been deposited.

\section{STRUCTURE}

The structures of more than 40 molecules containing the acetylenic group have been determined by spectroscopy and electron diffraction. ${ }^{8}$ Disregarding the exceptionally short $\mathrm{C} \equiv \mathrm{C}$ distance in fluoroacetylene ${ }^{9}\left(r_{\mathrm{s}}=119.8 \pm 0.3 \mathrm{pm}\right)$, the $\mathrm{C} \equiv \mathrm{C}$ bond distance appears to be almost unaffected by the substituents $\left(r_{\mathrm{s}}=120.4 \pm 0.3\right.$ and $120.9 \pm 0.2 \mathrm{pm}$ in chloroacetylene ${ }^{9}$ and tertbutylacetylene,${ }^{10}$ respectively). In spite of the experimental uncertainties, the expected trend of sigma electron donating substituents to lengthen and of sigma electron accepting substituents to shorten the $\mathrm{C} \equiv \mathrm{C}$ bond is observed. For MDA, with two conjugated $\mathrm{C} \equiv \mathrm{C}$ bonds, one would

* Microwave Data Center, Molecular Spectroscopy Section, National Bureau of Standards, Washington D. C. 20234, U.S.A. expect much smaller effects upon substitution, but the $\mathrm{C} \equiv \mathrm{C}$ bond adjacent to the methyl group to be the longest.

$\mathrm{Ab}$ initio calculations. The fully optimized structures for 1,3-butadiyne (diacetylene, DA) and MDA were calculated by $a b$ initio gradient calculations.

The calculations were carried out using the computer program MOLFORC ${ }^{11}$ in which the energy gradient with respect to the nuclear coordinates is calculated for a single-determinant SCF wavefunction and the molecular geometry determined by the force relaxation method. ${ }^{12}$ This program is based on the program MOLECULE ${ }^{13}$ which solves the Roothaan-Hall equations for a basis set of contracted gaussian functions.

The basis set applied was the $(7,3) \rightarrow\langle 4,2\rangle$ basis of Roos and Siegbahn ${ }^{14}$ for the carbon atoms, and Huzinagas' (4) $\rightarrow\langle 2\rangle^{15}$ basis set scaled by the factor 1.2 for the hydrogen atoms. ${ }^{16}$ This basis set is widely used and is expected to 
give consistent descriptions of molecular geometries, with the CC triple bonds about $0.02 \AA$ too short.

For DA the calculations were carried out starting with $C_{2 v}$ and $C_{2 h}$ symmetries. In both cases the calculations converged to the linear form. The geometry of MDA was optimized without symmetry restrictions. However, the geometry converged to the linear $C_{3 v}^{*}$ form with almost equal acetylenic bond lengths. In Table 2 the results from the $a b$ initio calculations are compared with the experimental structure.

$\mathbf{r}_{o}$-structure. The main problem in determining the structure of MDA is caused by the absence of experimental data for ${ }^{13} \mathrm{C}$ isotopic substitution of the central carbon atom $\left(\mathrm{C}_{3}\right)$. Hence, the (small) coordinate for $\mathrm{C}_{3}$ has to be determined by the first moment equations which are not suited at all for small coordinates. The $r_{\mathrm{o}}$-structure, given in Table 2, was derived by a least squares procedure using all the experimental moments of inertia for MDA and the isotopically substituted molecules (Table 3). The standard deviation of the fit, being five times larger than the experimental errors, indicates large zero-point vibrational effects. However, attempts to include bond shortening

Table 3. Experimental values for the moments of inertia with standard errors for 1,3-pentadiyne.

\begin{tabular}{|c|c|}
\hline Molecule & $I_{\mathrm{b}}^{\circ}\left[\mathrm{u} \AA^{2}\right]^{a}$ \\
\hline 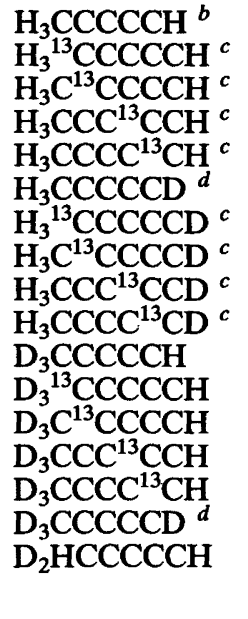 & $\begin{array}{l}248.25260(13) \\
254.897(6) \\
249.532(6) \\
250.317(6) \\
255.214(6) \\
261.8853(27) \\
268.829(7) \\
263.298(7) \\
263.790(7) \\
268.550(7) \\
275.43314(6) \\
281.40975(14) \\
276.4270(4) \\
277.8966(5) \\
283.11945(13) \\
290.078(3) \\
266.07615(29) \\
{\left[I_{\mathrm{c}}^{\circ}=267.2114(3)\right]}\end{array}$ \\
\hline
\end{tabular}

${ }^{a}$ Conversion factor: I B=505379.1 MHz u $\AA^{2} .1$ u $\AA^{2}=1.66053110^{-47} \mathrm{~kg} \mathrm{~m}^{2}$. ${ }^{b}$ From Ref. 3. ${ }^{c}$ From Ref. 2. ${ }^{d}$ From Ref. 1. upon deuteration in the refinement leads to large correlation between the structural parameters and unreasonable values for $r_{\mathrm{CH}}-r_{\mathrm{CD}}$.

$\mathbf{r}_{m}$-structure. It is possible to overcome the apparent inconsistency between the experimental moments of inertia and the structure parameters. This can be accomplished by assuming a functional mass dependence of the vibrational contribution to the effective moments of inertia. ${ }^{17}$ As suggested by Watson, ${ }^{17}$ a functional relationship of the form:

$$
\begin{aligned}
& I^{\circ}=I^{\prime}\left(r_{\mathrm{m}}\right)+\varepsilon_{1}+\varepsilon_{2} \\
& \varepsilon_{1}=I^{\prime}\left(r_{\mathrm{m}}\right)^{1 / 2} \\
& \varepsilon_{2}=\left[\frac{m_{1}^{3} m_{2}^{3} m_{3}^{3} m_{\mathrm{N}}^{3}}{M^{3} I_{\mathrm{a}}^{\prime} I_{\mathrm{b}}^{\prime} I_{\mathrm{c}}^{\prime}}\right]^{1 / 2(3 N-6)}
\end{aligned}
$$

should give (1) internal consistency between experimental data and structure parameters and (2) a better approximation to the equilibrium structure. As seen, both $\varepsilon_{1}$ and $\varepsilon_{2}$ are (as required) ${ }^{18}$ homogeneous functions of degree $1 / 2$ in the mass. Though this definition of the $r_{\mathrm{m}}$-structure differs from that originally given by Watson, ${ }^{18}$ the same labeling of the structure has been used in order to indicate the mass dependence.

In Table 2 are given the results of a least squares refinement where all experimental data (also $I_{c}$ for MDA-5,5- $d_{2}$ ) have been fitted to the above expression. In view of the uncertainties in the $r_{\mathrm{o}}$-structure, the differences in the $r_{\mathrm{m}^{-}}$and $r_{\mathrm{o}}$-structures are insignificant. However, the parameters in the $r_{\mathrm{m}}$-structure are far less correlated than in the $r_{\mathrm{o}}$-structure and all the moments of inertia are reproduced within their experimental uncertainties.

$\mathbf{r}_{s}$-structure. Five of the total of eight substitution coordinates in MDA can readily be determined from the Kraitchman equations ${ }^{19}$ using either MDA, MDA-1- $d$ or MDA-5,5,5- $d_{3}$ as the parent molecule. The two methyl group coordinates, the off-axis distances $(x)$ and the along the axis coordinate $(\mathrm{z})$, for the hydrogen atoms can be determined with either MDA or MDA-5,5,5$d_{3}$ as the parent molecule. This can be accomplished by combining the Kraitchman equations for multiple substitution: 


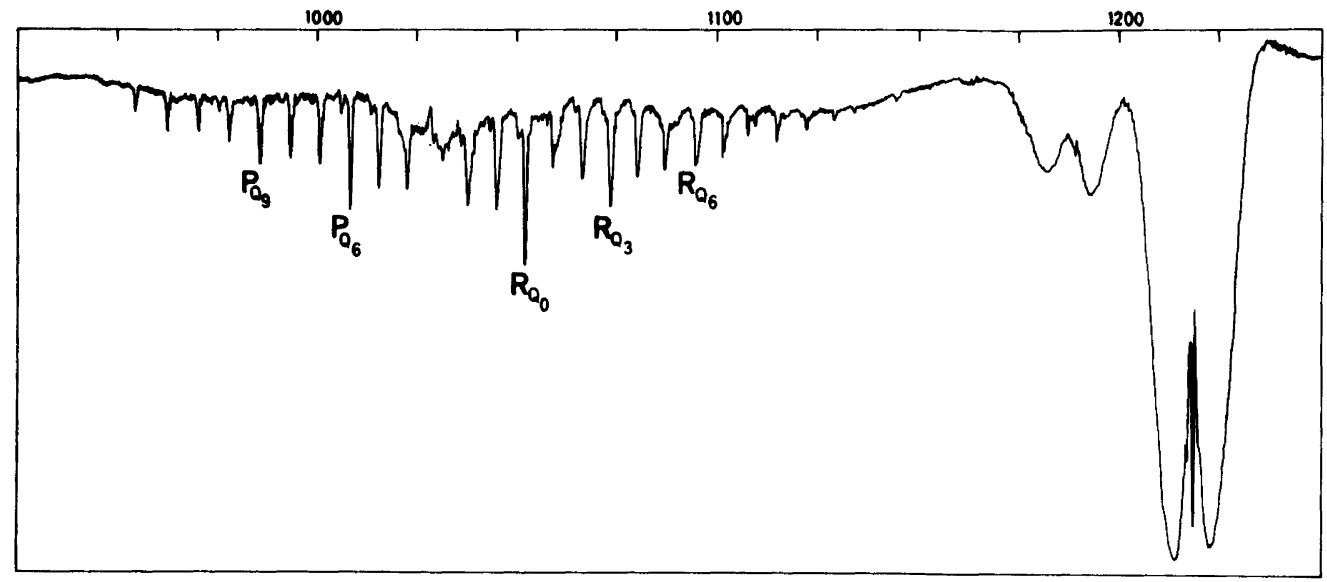

Fig. 1. Infrared spectrum in the region $1250-925 \mathrm{~cm}^{-1}$ of 1,3-pentadiyne-5,5,5- $d_{3}$ (resolution 0.25 $\mathrm{cm}^{-1}$ ) showing the $v_{6}\left(a_{1}\right)$ band at $1025 \mathrm{~cm}^{-1}$, the $v_{9}(e)$ band at $1046 \mathrm{~cm}^{-1}$, the $v_{5}\left(a_{1}\right)$ band at $1187 \mathrm{~cm}^{-1}$ and the $2 v_{11}\left(a_{1}+e\right)$ band at $1218 \mathrm{~cm}^{-1}$.

$$
\begin{aligned}
& \mathrm{CH}_{3} \rightarrow \mathrm{CD}_{3}: \\
& \Delta I_{3}=\frac{3}{2} \Delta m x^{2}+\mu_{3} z^{2}, \mu_{3}=\frac{3 \Delta m M}{M+3 \Delta m} \\
& \mathrm{CH}_{3} \rightarrow \mathrm{CD}_{2} \mathrm{H}: \\
& \Delta I_{2}=\frac{1}{4} \mu_{2} x^{2}+\mu_{2} z^{2}, \mu_{2}=\frac{2 \Delta m M}{m+2 \Delta m} \\
& \mathrm{CD}_{3} \rightarrow \mathrm{CH}_{3}: \\
& \Delta I_{3}=\frac{3}{2} \Delta m x^{2}+\mu_{3} z^{2}, \mu_{3}=\frac{3 \Delta m M}{M+3 \Delta m} \\
& \mathrm{CD}_{3} \rightarrow \mathrm{CD}_{2} \mathrm{H}: \\
& \Delta I_{1}=\mu_{1} x^{2}+\mu_{1} z^{2}, \mu_{1}=\frac{\Delta m M}{M+\Delta m}
\end{aligned}
$$

In both cases (the determinant being $c a .1 .5$ ) the equations are easily solved. If the $x$-coordinate for the methyl hydrogen is transferred from MDA or MDA-5,5,5- $d_{3}$ to MDA-1- $d$, then the corresponding $z$-coordinate in MDA-1- $d$ can be found from the equation for the $\mathrm{CH}_{3} \rightarrow \mathrm{CD}_{3}$ substitution.

The remaining coordinate for the central carbon atom has to be determined from the first moment equations. This coordinate was found to be $+0.070 \pm 0.004,+0.204 \pm 0.001$ and $+0.013 \pm 0.005 \AA$ in MDA, MDA-5,5,5- $d_{3}$ and MDA-1- $d$, respectively. The derived $r_{\mathrm{s}}$-structure is compared with the $r_{\mathrm{o}}$ - and the $r_{\mathrm{m}}$-structure in Table 2.

\section{VIBRATIONAL SPECTRA}

Coriolis coupling constants in $M D A-5,5,5-\mathrm{d}_{3}$. The Coriolis coupling constants for the degenerate methyl vibrations have been determined for MDA. ${ }^{5}$ For MDA-5,5,5- $d_{3}$ the corresponding vibrational bands $\left(v_{8}, v_{9}\right.$ and $\left.v_{10}\right)$ should all show resolvable rotational fine structure. However, due to the limited amount of sample, the $v_{10}$ band at $c a .850 \mathrm{~cm}^{-1}$ was too weak to be observed with any confidence. The $v_{8}$ band at $c a .2250 \mathrm{~cm}^{-1}$ was partly obscured by the stronger $v_{2}\left(a_{1}\right)$ band at $2258 \mathrm{~cm}^{-1}$ and it was not possible to determine the band centre. Hence, only the $v_{9}$ band at $c a$. $1050 \mathrm{~cm}^{-1}$ was studied in detail.

The $Q$-branches of the sub-bands that arise from the $\Delta K= \pm 1, \Delta J=0$ transitions are given by the expression: ${ }^{20}$

$$
\begin{aligned}
& v_{9}^{K}=v_{9}+\left[A^{\prime}\left(1-\zeta_{9}\right)^{2}-B^{\prime}\right] \pm 2\left[A^{\prime}\left(1-\zeta_{9}\right)-B^{\prime}\right] K \\
& +\left[\left(A^{\prime}-A^{\prime \prime}\right)-\left(B^{\prime}-B^{\prime \prime}\right)\right] K^{2}
\end{aligned}
$$

Using the experimental value for $B^{\prime \prime}$, a value for $A^{\prime \prime}$ calculated from the $r_{\mathrm{o}}$-structure $\left(A^{\prime \prime}=2.590\right.$ $\mathrm{cm}^{-1}$ ) and further assuming that $B^{\prime}=B^{\prime \prime}, \zeta_{9}$ and $v_{9}$ can be calculated from the above equation.

As shown in Fig. 1, the $v_{9}$ band is overlapped by the weak $v_{6}\left(a_{1}\right)$ band at ca. $1025 \mathrm{~cm}^{-1}$. The intensity distribution among the sub-bands permits a choice of either $c a .1051$ or $c a .1031 \mathrm{~cm}^{-1}$ as the $K=0 \rightarrow 1$ transition. That the former is prob- 
Table 4. $v_{9}$ of 1,3-pentadiyne-5,5,5- $d_{3}$.

\begin{tabular}{|c|c|c|c|c|}
\hline \multirow{2}{*}{$\begin{array}{l}K \text {-Value in } \\
\text { sub-bands }\end{array}$} & \multicolumn{2}{|l|}{$\boldsymbol{P}_{\mathbf{Q}_{\mathbf{K}}}$} & \multicolumn{2}{|l|}{$\boldsymbol{R}_{\mathbf{Q}_{\mathrm{K}}}$} \\
\hline & $\begin{array}{l}\text { Obs. } \\
{\left[\mathrm{cm}^{-1}\right]}\end{array}$ & Obs.-calc. & $\begin{array}{l}\text { Obs. } \\
{\left[\mathrm{cm}^{-1}\right]}\end{array}$ & Obs.-calc. \\
\hline $\begin{array}{r}0 \\
1 \\
2 \\
3 \\
4 \\
5 \\
6 \\
7 \\
8 \\
9 \\
10 \\
11 \\
12 \\
13 \\
14\end{array}$ & $\begin{array}{r}1044.58 \\
1037.34 \\
\\
1021.91 \\
1014.68 \\
1007.44 \\
1000.21 \\
992.49 \\
985.26 \\
977.54 \\
969.83 \\
962.11 \\
954.39 \\
946.68\end{array}$ & $\begin{array}{r}0.26 \\
0.31 \\
\\
-0.43 \\
-0.26 \\
-0.07 \\
0.17 \\
-0.04 \\
0.27 \\
0.13 \\
0.03 \\
-0.04 \\
-0.07 \\
-0.06\end{array}$ & $\begin{array}{l}1051.33 \\
1058.56 \\
1066.28 \\
1073.03 \\
1080.26 \\
1087.01 \\
1094.73 \\
1101.48 \\
\\
1114.99 \\
1122.22 \\
1128.97\end{array}$ & $\begin{array}{r}-0.24 \\
-0.23 \\
0.31 \\
-0.09 \\
0.03 \\
-0.30 \\
0.39 \\
0.13 \\
\\
-0.25 \\
0.08 \\
-0.26\end{array}$ \\
\hline & \multicolumn{2}{|c|}{$\begin{array}{l}\nu_{9}^{\circ}=1046.4 \pm 0.3 \mathrm{~cm}^{-1} a \\
\zeta_{9}=-0.41 \pm 0.02\end{array}$} & & \\
\hline
\end{tabular}

${ }^{a}$ Estimated from an uncertainty in $\mathrm{A}^{\prime}$ of $5 \%$.

ably the correct assignment is supported by a solution/vapour shift of $20 \mathrm{~cm}^{-1}$ for this type of vibration. ${ }^{5}$

The observed $Q$-branches are listed in Table 4 together with the derived parameters. A standard error of $0.3 \mathrm{~cm}^{-1}$ for the fit is rather larger in view of the resolution used in obtaining the spectrum (ca. $0.25 \mathrm{~cm}^{-1}$ ), but a secord order Coriolis coupling with $v_{5}$ and/or $v_{6}$ is a plausible explanation for this.

Vibrational fundamentals. From the two previous investigations of the vibrational spectra of MDA $^{4,5}$ and the deuterated molecules ${ }^{5}$ a reliable assignment of most of the fundamental modes of vibration is known. Three obvious cases of Fermi resonance are observed. The $v_{3}$ mode

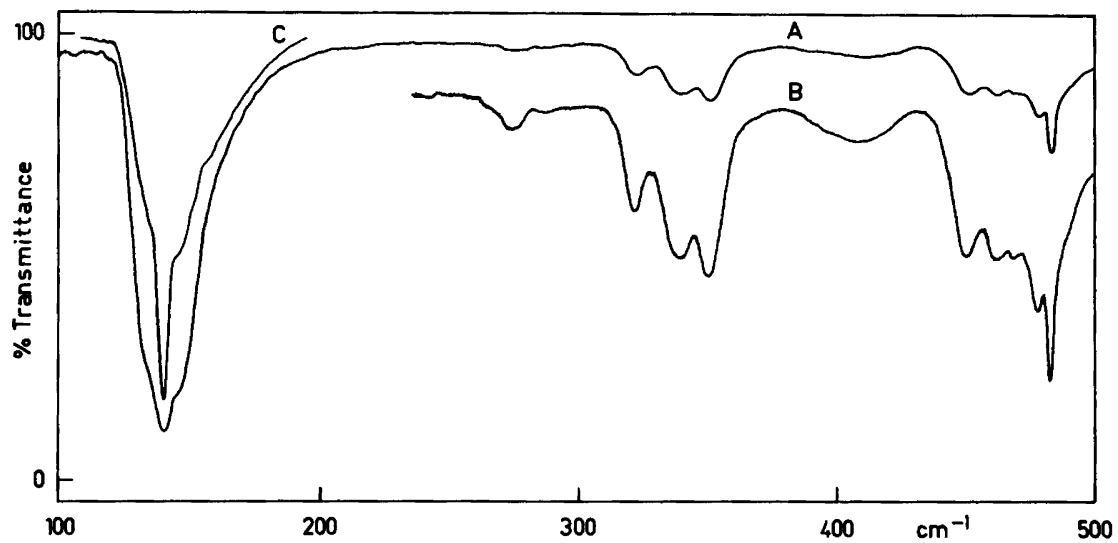

Fig. 2. Far infrared spectrum of 1,3-pentadiyne as a vapour. (A) $50 \mathrm{Torr}, 20 \mathrm{~cm}$, (B) scale expansion of $A$ and (C) 10 Torr, $20 \mathrm{~cm}$. 
Table 5. Far infrared vapour phase spectral data for 1,3-pentadiyne.

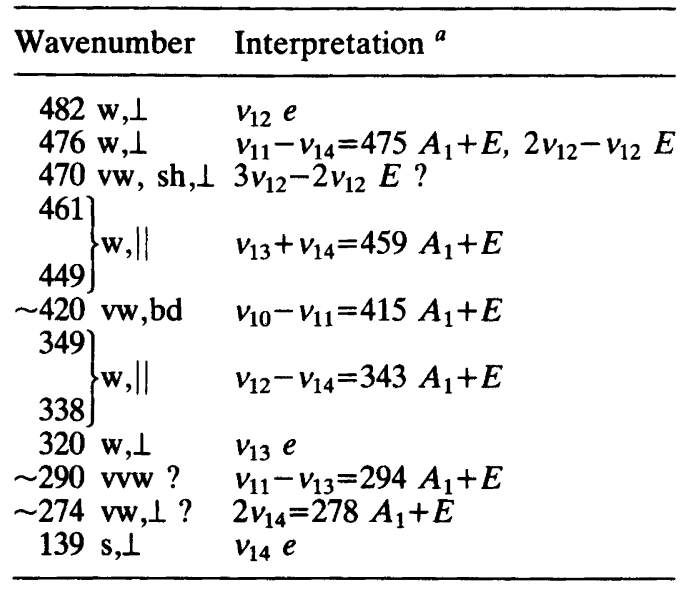

${ }^{a}$ Abbreviations: s: strong; v: very; w: weak; $\|$ : parallel; $\perp$ : perpendicular; bd: broad; sh: shoulder.
( $\mathrm{C} \equiv \mathrm{C}$ asym. stretch) in MDA and MDA-1- $d$ is in resonance with the $2 v_{6}$ mode. ${ }^{4}$ Assuming an anharmonicity $X_{6}=0.001$, estimated from the 1-halogenated 1,5-pentadiynes, ${ }^{5}$ corrections were made for the $v_{3}$ fundamentals. Fermi resonance is also observed between $v_{2}$ (三C-D stretch) and $v_{4}+v_{7}$ in MDA- $d_{4}$. Since no frequency shift is expected for this mode in going from MDA-1-d to $\mathrm{MDA}-d_{4}, v_{2}$ is assumed to have the same frequency in these two molecules. In a few cases the fundamentals above $400 \mathrm{~cm}^{-1}$ have not been observed in the vapour phase. When possible, these fundamental frequencies were corrected by transferring the corresponding liquid/vapour shifts observed for the other isotopic species.

The two lowest degenerate fundamentals, $v_{13}$ and $v_{14}$, have not been observed in the vapour phase previously. In Fig. 2 the far IR vapour phase spectrum of MDA is shown, while the wave numbers of the observed bands are given in

Table 6. Internal valence symmetry coordinates for 1,3-pentadiyne with reference to Fig. 3.

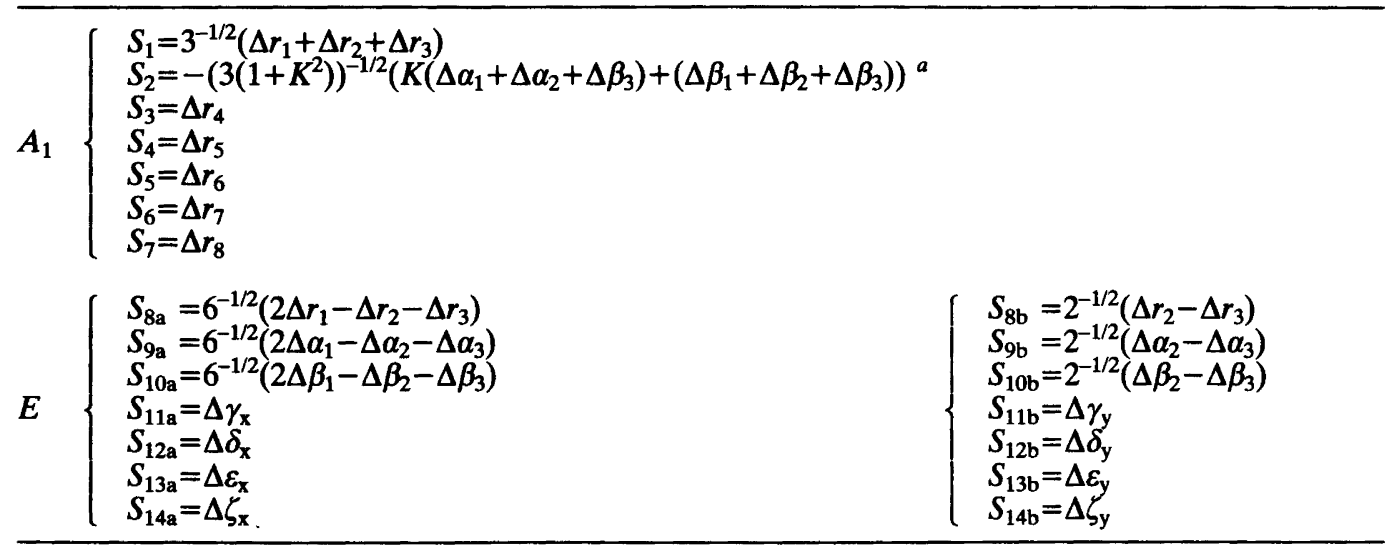

a The angle redundancy has been removed: $\Delta \alpha=K \Delta \beta, K=3 \sin \beta \cos \beta / \sin \alpha$.

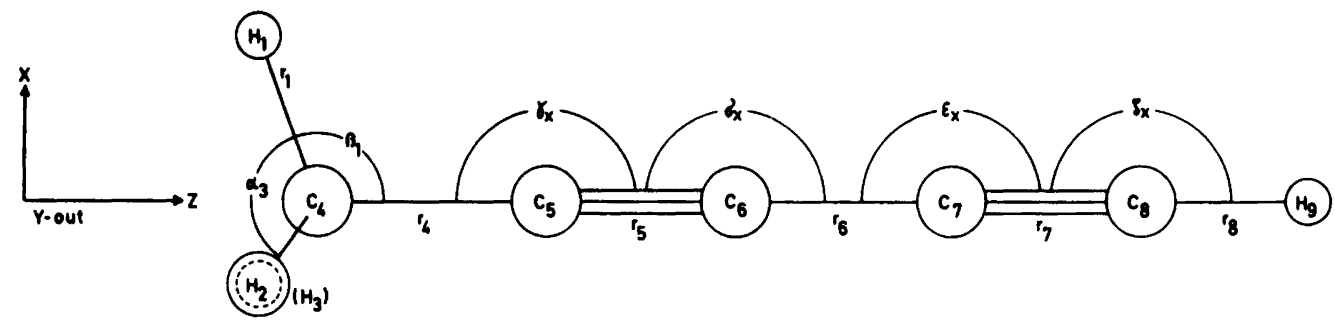

Fig. 3. Valence coordinates for 1,3-pentadiyne. 
Table 7. Force constants and standard errors, in symmetry coordinate representation, for 1,3 -pentadiyne. Stretching force constants in mdyn $/ \AA$, bending force constants in $\mathrm{mdyn} \AA / \mathrm{rad}^{2}$ and stretch-bend interactions in mdyn/rad.

\begin{tabular}{llllllll}
\hline & $F^{a}$ & $\sigma(\mathrm{F})$ & $R, B, C^{b}$ & & $F$ & $\sigma(\mathrm{F})$ & $R, B, C$ \\
\hline$F_{1,1}$ & 4.953 & 0.034 & 4.96 & $F_{8,8}$ & 4.777 & 0.032 & 4.71 \\
$F_{1,2}$ & 0 & & 0 & $F_{8,9}$ & 0 & & 0 \\
$F_{2,2}$ & 0.5866 & 0.0070 & 0.51 & $F_{8,10}$ & 0 & & 0 \\
$F_{2,3}$ & -0.426 & 0.037 & -0.34 & $F_{9,9}$ & 0.559 & 0.014 & 0.53 \\
$F_{3,3}$ & 5.54 & 0.23 & 5.57 & $F_{9,10}$ & -0.01 & 0.03 & 0 \\
$F_{3,4}$ & 0.34 & - & 0 & $F_{10,10}$ & 0.639 & 0.025 & 0.68 \\
$F_{4,4}$ & 15.67 & 0.21 & 15.17 & $F_{10,11}$ & -0.062 & 0.019 & 0 \\
$F_{4,5}$ & 1.14 & - & 0 & $F_{11,11}$ & 0.316 & 0.029 & 0.42 \\
$F_{4,6}$ & 0 & & 0 & $F_{11,12}$ & 0.071 & 0.017 & 0 \\
$F_{5,5}$ & 7.23 & 0.35 & 7.52 & $F_{12,12}$ & 0.314 & 0.021 & 0.38 \\
$F_{5,6}$ & 0.72 & 0.30 & 0 & $F_{12,13}$ & 0.015 & 0.015 & 0 \\
$F_{6,6}$ & 15.14 & 0.31 & 14.39 & $F_{13,13}$ & 0.348 & 0.036 & 0.37 \\
$F_{6,7}$ & 0 & & 0 & $F_{13,14}$ & 0.127 & 0.009 & 0 \\
$F_{7,7}$ & 5.979 & 0.047 & 5.95 & $F_{14,14}$ & 0.212 & 0.002 & 0.17 \\
\hline
\end{tabular}

${ }^{a} 1 \mathrm{mdyn} / \AA=10^{2} \mathrm{~N} / \mathrm{m}, 1 \mathrm{mdyn} \AA / \mathrm{rad}^{2}=10^{-18} \mathrm{Nm} / \mathrm{rad}^{2}, 1 \mathrm{mdyn} / \mathrm{rad}=10^{-8} \mathrm{~N} / \mathrm{rad}{ }^{b}$ Rogstad, Benestad and Cyvin (Ref. 6) assumed tetrahedral angles and the following geometry: $r_{1}=110.0, r_{4}=145.8, r_{5}=r_{7}=120.7, r_{6}=137.5$ and $r_{8}=105.7 \mathrm{pm}$.

Table 5 with the proposed assignment. Apart from the possible hot-band progression of the $v_{12}$ mode at $482 \mathrm{~cm}^{-1}$, the rest of the observed bands are unambiguously assigned.

\section{FORCE CONSTANT CALCULATION}

In Table 6, internal valence symmetry coordinates are given which can be interpreted by reference to Fig. 3. The molecular geometry is taken as the $r_{\mathrm{s}}$-structure given in Table 2 and the atomic masses are based upon the ${ }^{12} \mathrm{C}$-scale.

All the available data on vibrational frequencies, centrifugal distortion constants and Coriolis coupling constants for the various isotopic species have been used for calculating the force field. Since anharmonicity constants for MDA are unknown, and the corrections to harmonic frequencies would have to be guessed, the anharmonic vapour phase frequencies have been used whenever possible.

The weights, $w_{i}$, assigned to the data were calculated from the equation: $w_{\mathrm{i}}=1 / \sigma_{\mathrm{i}}^{2}$, where $\sigma_{\mathrm{i}}$ is the estimated probable error in the $i$ th datum. $\sigma_{i}$ has been taken equal to 0.01 (frequency), but not less than $5 \mathrm{~cm}^{-1}$ for the vibrational frequencies. For the $\zeta$ constants, an uncertainty of 0.05 has been allowed to account for unknown anhar-
Table 8. 1,3-Pentadiyne; observed data and uncertainties $(\sigma)$, calculated data and errors $(\varepsilon)$. Fundamental modes of vibration in wavenumbers, $D_{\mathrm{J}}$ in $\mathrm{Hz}, D_{\mathrm{JK}}$ in $\mathrm{kHz}, \zeta$-constants dimensionless and $l$-type doubling constants, $q_{i}$, in MHz.

\begin{tabular}{lrrrr}
\hline & Obs. & $\sigma$ & \multicolumn{1}{c}{ Calc. } & \multicolumn{1}{c}{$\varepsilon$} \\
\hline $\mathrm{H}_{3} \mathrm{CCCCCH}^{a}$ & & & & \\
$v_{1}$ & 3333 & 33 & 3343 & -10 \\
$v_{2}$ & 2935 & 29 & 2938 & -3 \\
$v_{3}$ & $22577^{b}$ & 23 & 2261 & -4 \\
$v_{4}$ & 2079 & 21 & 2082 & -3 \\
$v_{5}$ & 1385 & 14 & 1394 & 1 \\
$v_{6}$ & 1150 & 12 & 1170 & -20 \\
$v_{7}$ & 696 & 7 & 687 & -9 \\
& & & & \\
$v_{8}$ & 2977 & 30 & 2996 & -19 \\
$v_{9}$ & 1446 & 14 & 1449 & -3 \\
$v_{10}$ & 1029 & 10 & 1031 & -2 \\
$v_{11}$ & 614 & 6 & 616 & -2 \\
$v_{12}$ & 484 & 5 & 486 & -2 \\
$v_{13}$ & 320 & 5 & 320 & 0 \\
$v_{14}$ & 139 & 5 & 138 & 1 \\
$\zeta_{8}$ & & & & \\
$\zeta_{9}$ & 0.08 & 0.05 & 0.15 & -0.07 \\
$\zeta_{10}$ & -0.34 & 0.05 & -0.37 & 0.03 \\
$\zeta_{13}$ & 0.40 & 0.05 & 0.36 & 0.04 \\
$\zeta_{14}$ & 0.94 & 0.05 & 0.93 & 0.01 \\
& 0.96 & 0.05 & 0.96 & 0.
\end{tabular}




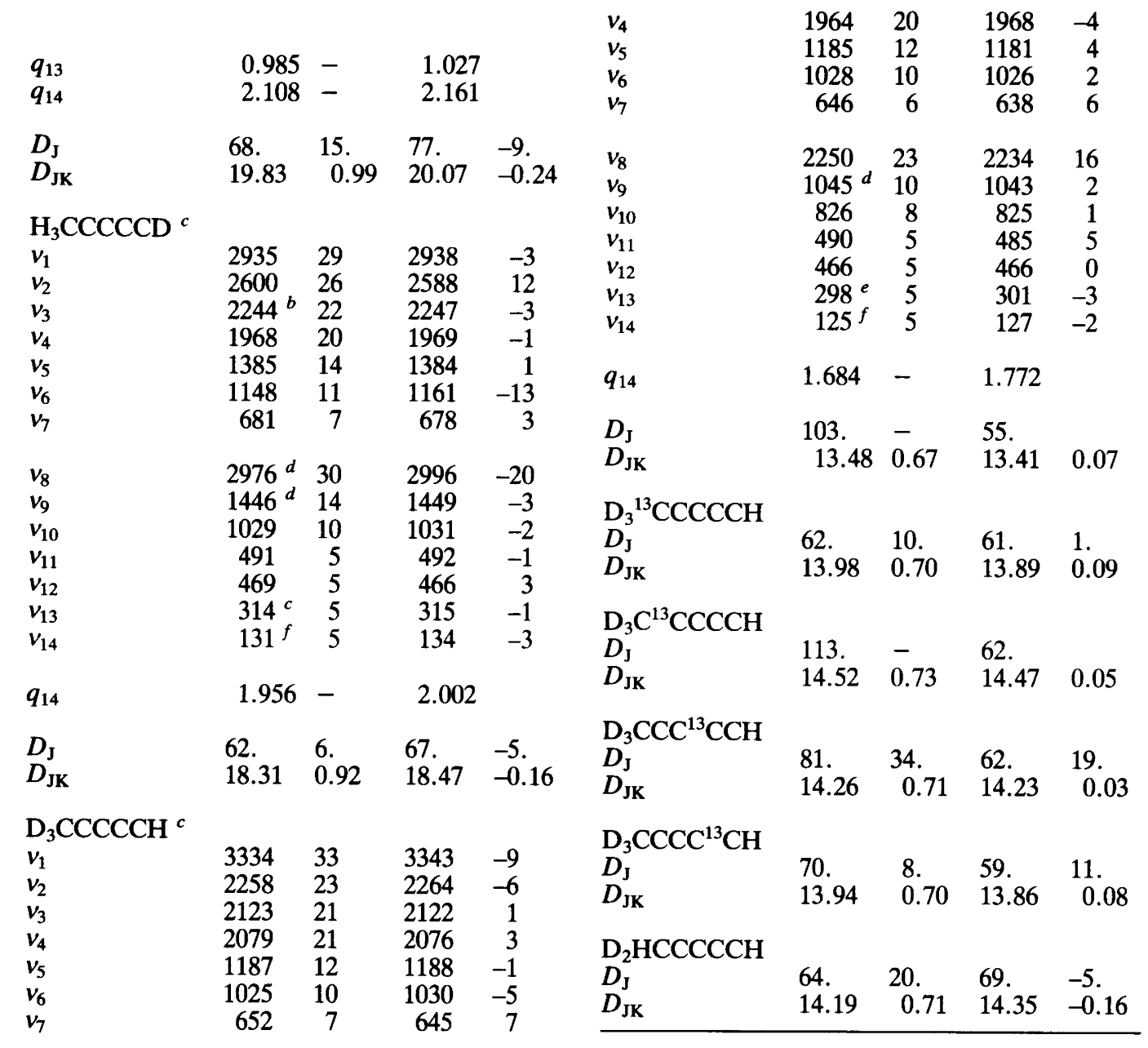

${ }^{a}$ Vibrational frequencies from Ref. 5, Coriolis coupling constants from Ref. 5 and Ref. 3, l-type doubling constants from Ref. 2 and distortion constants from Ref. 1. ${ }^{b}$ Corrected for Fermi resonance. ${ }^{c}$ Vibrational frequencies from Ref. 4 , distortion constants and $l$-type doubling constants from Ref. $1 .{ }^{d}$ Corrected from $\left(R_{\mathrm{Q}_{0}}\right)$ to center frequency. ' Solution data from Raman spectrum. ${ }^{f}$ Corrected for liquid/vapour frequency shift.

$\begin{array}{llllll}\zeta_{9} & & -0.41 & 0.05 & -0.44 & 0.03\end{array}$

$q_{14} \quad 1.804-\quad 1.913$

$\begin{array}{lllll}D_{\mathrm{J}} & 70 . & 5 . & 63 . & 7 .\end{array}$

$\begin{array}{lllll}D_{\mathrm{JK}} & 14.55 & 0.73 & 14.51 & 0.04\end{array}$

$\mathrm{D}_{3} \mathrm{CCCCCD}^{c}$

$\begin{array}{llllr}v_{1} & 2600^{b} & 26 & 2588 & 12 \\ v_{2} & 2250 & 23 & 2251 & -1 \\ v_{3} & 2121 & 21 & 2118 & 3\end{array}$

monicity effects. The $\sigma\left(D_{\mathrm{J}}\right)$ have been taken as two times the standard error on these constants (poorly determined) and the $\sigma\left(D_{\mathrm{JK}}\right)$ have been taken as $5 \%$ of their values to allow for anharmonicity.

The results of a convergence to a 23 parameter force field are given in Table 7 . In the $A_{1}$ species it was found necessary to constrain the 
stretch-stretch interaction constants $F_{3,4}$ and $F_{4,5}$ to their values in 2,4-hexadiyne (dimethyldiacetylene). ${ }^{21}$ The $E$ species force constants are more reliably fixed, primarily due to the availability of $D_{\mathrm{jk}}$ for a number of ${ }^{13} \mathrm{C}$ isotopic substituted molecules. The reproduction of the observed data for all isotopes of MDA, calculated using the force field of Table 7 , is given in Table 8.

Acknowledgements. The authors are grateful to the Department of Chemical Physics, University of Copenhagen, for the use of their microwave spectrometer. Financial support from the Norwegian Research Council for Science and the Humanities is acknowledged.

\section{REFERENCES}

1. Heath, G. A., Thomas, L. F., Sherrad, E. I. and Sheridan, J. Discuss. Faraday Soc. 19 (1955) 38.

2. Thomas, L. F., Heeks, J. S. and Sheridan, J. Arch. Sci. 10 (1957) 180.

3. Maier, M. Thesis, University of Sussex, Sussex 1977.

4. Lamotte, J., Lavalley, J. C. and Romanet, R. J. Chim. Phys. Phys. Chim. Biol. 70 (1973) 1077.

5. Benestad, L., Augdahl, E. and KlosterJensen, E. Spectrochim. Acta A 31 (1975) 1329.

6. Rogstad, A., Benestad, L. and Cyvin, S. J. J. Mol. Struct. 23 (1974) 265.

7. Maier, J. P., Marthaler, O. and KlosterJensen, E. J. Chem. Phys. 72 (1980) 701.

8. Callomon, J. H., Hirota, E., Kuchitsu, K., Lafferty, W. J., Maki, A. G. and Pote, C. S. Structure Data of Free Polyatomic Molecules, Springer, Berlin 1976.

9. Tyler, J. K. and Sheridan, J. Trans. Faraday Soc. 59 (1963) 2661.

10. Nugent, L. J., Mann, D. E. and Lide, D. R. J. Chem. Phys. 36 (1962) 965.

11. Sæbø, S. The Program System MOLFORC, Program Manual, Department of Chemistry, University of Oslo, Oslo 1979.

12. Pulay, P. Mol. Phys. 17 (1969) 197.

13. Almlöf, J. The Program System MOLECULE, USIP Report 74-29, University of Stockholm, Stockholm 1974.

14. Roos, B. and Siegbahn, P. Theor. Chim. Acta 17 (1970) 209.

15. Huzinaga, S. J. Chem. Phys. 42 (1965) 1293.

16. Dunning, T. H., Jr., J. Chem. Phys. 53 (1970) 2823.
17. Watson, J. K. G. 5th International Seminar on High Resolution Infrared Spectroscopy, Prague 1978.

18. Watson, J. K. G. J. Mol. Spectrosc. 48 (1973) 479.

19. Kraitchman, J. Am. J. Phys. 21 (1953) 17.

20. Thomas, R. K. and Thompson, H. W. Spectrochim. Acta A 24 (1968) 1337.

21. Nielsen, C. J. To be published.

Received August 30, 1982. 